\title{
Angular Distribution of Nonlinear Harmonic Generation in Helical Undulators: a comparison between experiments and theory
}

\author{
E. Allaria ${ }^{1}$, G. De Ninno ${ }^{1,2}$, G. Geloni ${ }^{3}$, C. Spezzani ${ }^{1}$ \\ 1. Sincrotrone Trieste, S.S. $14 \mathrm{~km}$ 163.5, Basovizza (Ts), Italy \\ 2. Physics Department, \\ Nova Gorica University, Nova Gorica, Slovenia \\ 3. European XFEL GmbH, \\ Albert-Einstein-Ring 19, 22761 Hamburg, Germany
}

(Dated: March 2, 2022)

\begin{abstract}
Using harmonic emission from circularly polarized undulator is a procedure that is normally employed on synchrotron beamlines in order to extend the covered spectral range. A similar capability is likewise beneficial for next generation free-electron lasers. In this paper, we perform a first quantitative experimental analysis of the angular distribution of free-electron laser harmonic emission from helical undulators. Experimental results are compared to those obtained by means of a theoretical model based on the paraxial solution of Maxwell's equations.
\end{abstract}

PACS numbers:

\section{INTRODUCTION}

Variable polarization is one of the most attractive features of light sources based on electron beams propagating through APPLE-type undulators.

Such a feature is particularly attractive for those applications aiming to investigate the local symmetry of a given system, especially when the symmetry can be ascribed to the lattice geometry (as in the case of crystals), to the chirality of a molecule or to a possible local atomic magnetic moment. In fact, several spectroscopic methods rely on the opportunity to chose a well defined polarization state of the light. Spin [1] and angular resolved photo-electron spectroscopy, x-ray linear and circular dichroism or resonant scattering of polarized $\mathrm{x}$ rays [2, 3] are only few examples. For this reason, light polarization-dependent spectroscopy using standard synchrotron radiation has become a very powerful tool to study the electronic and magnetic properties of matter [4, 5].

Thanks to a dramatic increase of photon peakbrilliance with respect to conventional sources, as well as to the possibility of controlling both the temporal duration and the spectral bandwidth of the produced light pulses, free-electron lasers (FELs) will allow completely new studies in the above mentioned research fields [6]. Such studies will also take advantage of well-defined and easily tunable light polarization. For the preparation of the scientific case of single-pass FELs, a fundamental question concerns the possibility of generating significant photon flux at higher harmonics of the fundamental wavelength, while maintaining polarization ductility.

In a FEL, harmonic generation is driven by the electron-beam interaction with a radiation at a given fundamental wavelength, in the presence of the static and periodic magnetic field generated by an undulator [7 14]. Such interaction produces a significant modulation (called bunching) of the electron-beam density at the fundamental wavelength, and its harmonics. Harmonic bunching is the (nonlinear) source of FEL harmonic emission. The latter is orders of magnitudes stronger than the spontaneous incoherent emission and provides the possibility to extend the FEL tuning range towards short wavelengths.

In the simplest possible configuration, FEL harmonics are produced in the same undulator where radiation at the fundamental wavelength is generated. This method is the standard one when the FEL light is obtained from Self Amplified Spontaneous Emission (SASE) 15], and is normally referred to as nonlinear harmonic generation (NHG).

An alternative approach to NHG for producing coherent harmonic emission relies upon the bunching created in an undulator, called the modulator, for generating coherent emission in a subsequent undulator, called the radiator, tuned at one of the harmonics of the modulator [16, 17]. This technique is usually called coherent harmonic generation (CHG). The implementation of CHG requires the use of an external coherent source, acting as a "seed", which is normally provided by one of the harmonics of a Ti: sapphire laser. These harmonics can be produced either using a solid-state crystal, or a gas jet 18]. The seed-electron interaction in the modulator is necessary to initiate the bunching process. Bunching is produced at all (odd and even) harmonics. As a consequence, in the radiator, coherent emission may occur at any selected harmonics, no matter the radiator polarization. The situation changes when NHG is considered. It is well known that, in case of planar undulators, on-axis NHG occurs only at odd harmonics, while even harmonics are present only off-axis [7]. In [19] it has been experimentally demonstrated that, as in the case of spontaneous emission, the NHG signal generated by helical undulators is distributed off-axis. A quantitative estimate of the off-axis coherent harmonic flux is, however, still missing. This lack of information is due to the absence, 


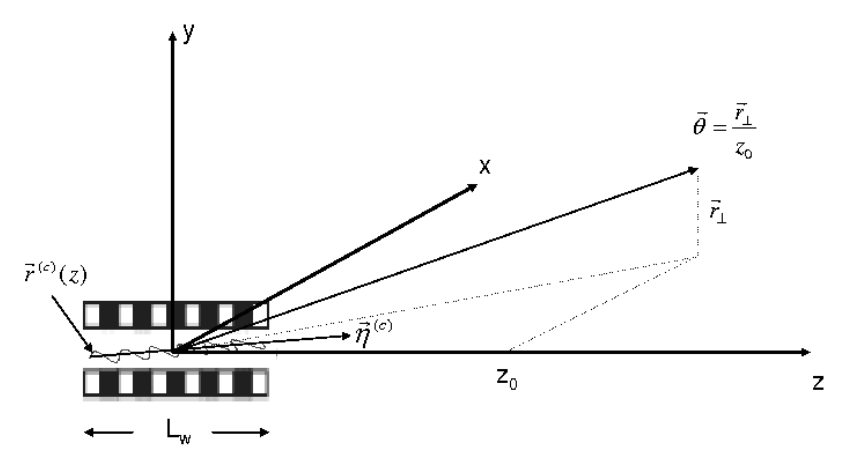

FIG. 1: Reference system and symbols used to develop the theoretical model.

so far, of an experimental characterization of the angular distribution of harmonic emission and, at the same time, to the difficulty of modelling the coherent emission from a modulated electron beam in the far zone. Recently, an analytical model has been proposed [20 23], relying on the paraxial solution of Maxwell's equations. Such a model confirms what has been found in [19], i.e. no on-axis NHG in helical undulators, and provide a quantitative prediction of the far-field angular harmonic distribution.

In this paper, we perform a first quantitative experimental analysis of the angular distribution of FEL harmonic radiation generated by a helical undulator. In particular, we characterize the off-axis emission in NHG configuration. Obtained results are used to benchmark the theoretical model proposed in 2023$]$.

The paper is structured as follows. In Section [II we briefly review the theoretical model and discuss the angular characteristics of the predicted far-field harmonic distribution. In Section III we present the experimental device we used to perform the measurements, i.e. the FEL installed at the Elettra storage-ring [25, 26]. In Section IV] experimental data are presented and compared to theoretical predictions. Finally, in Section $\nabla$ we draw conclusions and provide perspectives for future work.

\section{THEORETICAL FRAMEWORK}

The coherent emission in the far zone from modulated electron beams in planar or helical undulators can be studied by solving the paraxial Maxwell's equation for the electric field in the space-frequency domain [20 23].

Let us call the transverse electric field in the spacefrequency domain $\vec{E}_{\perp}\left(z, \vec{r}_{\perp}, \omega\right)$, where, as shown in Fig. 1. $\vec{r}_{\perp}=x \vec{e}_{x}+y \vec{e}_{y}$ identifies a point on a transverse plane at longitudinal position $z, \vec{e}_{x}$ and $\vec{e}_{y}$ being unit vectors in the transverse $x$ and $y$ directions.

The paraxial approximation is well suited for modelling the emission from ultra-relativistic electrons (see [21, 22]). It relies on the assumption that the elec- tric field envelope $\overrightarrow{\widetilde{E}}_{\perp}=\overrightarrow{\vec{E}}_{\perp} \exp [-i \omega z / c]$ does not vary much along $z$ on the scale of the reduced wavelength $\lambda=\lambda /(2 \pi)$. The wave equation for the slowly-varying envelope of the electric field is found to be

$$
\begin{aligned}
& \left(\nabla_{\perp}^{2}+\frac{2 i \omega}{c} \frac{\partial}{\partial z}\right)\left[\overrightarrow{\widetilde{E}}_{\perp}\left(z, \vec{r}_{\perp}, \omega\right)\right]= \\
& -4 \pi\left(\frac{i \omega}{c^{2}} \vec{j}_{\perp}-\vec{\nabla}_{\perp} \bar{\rho}\right) \exp \left[-\frac{i \omega z}{c}\right] .
\end{aligned}
$$

(where $c$ is the speed of light in vacuum). The right hand side is determined by the trajectory of the source electrons, and is written in terms of the Fourier transform of the transverse current density, $\vec{j}_{\perp}\left(z, \vec{r}_{\perp}, \omega\right)$, and of the charge density, $\bar{\rho}\left(z, \vec{r}_{\perp}, \omega\right)$, which will be considered as given macroscopic quantities. The Fourier transform of the charge density can be written as

$$
\bar{\rho}=-\widetilde{\rho}\left(z, \vec{r}_{\perp}-{\overrightarrow{r^{\prime}}}_{o \perp}(z), \omega\right) \exp \left[i \omega \frac{s_{o}(z)}{v_{o}}\right]
$$

where the minus sign on the right hand side is introduced for notational convenience. In the latter expression, the quantities ${\overrightarrow{r^{\prime}}}_{o \perp}(z), s_{o}(z)$ and $v_{o}$ are, respectively, the transverse position, the curvilinear abscissa and speed of a reference electron with Lorentz factor $\gamma$ that is injected on axis with no deflection and is guided by the undulator field. Such electron follows a trajectory ${\overrightarrow{r^{\prime}}}_{o \perp}(z)=r_{o x}^{\prime} \vec{e}_{x}+r_{o y}^{\prime} \vec{e}_{y}$, which assumes different forms, namely:

$$
{\overrightarrow{r^{\prime}}}_{o \perp}(z)=\frac{K}{\gamma k_{w}}\left[\left(\cos \left(k_{w} z\right)-1\right) \vec{e}_{x}+\sin \left(k_{w} z\right) \vec{e}_{y}\right]
$$

if the electron propagates through a helical undulator and

$$
{\overrightarrow{r^{\prime}}}_{o \perp}(z)=\frac{K}{\gamma k_{w}}\left(\cos \left(k_{w} z\right)-1\right) \vec{e}_{x}
$$

if the undulator is planar. Here $K=\lambda_{w} e H_{w} /\left(2 \pi m_{e} c^{2}\right)$ is the undulator parameter, $\lambda_{w}=2 \pi / k_{w}$ being the undulator period, $e$ the electron charge, $H_{w}$ the maximal modulus of the on-axis magnetic field of the undulator, and $m_{e}$ the rest mass of the electron. The corresponding velocity is given by $\vec{v}_{o \perp}(z)=v_{o x} \vec{e}_{x}+v_{o y} \vec{e}_{y}$.

Since we want to discuss an FEL process, $\widetilde{\rho}$ in Eq. (22) is a slowly varying function of $z$ on the wavelength scale and is peaked around the harmonics of the fundamental (i.e. at frequencies $h \omega_{r}=2 h k_{w} c \bar{\gamma}_{z}^{2}, \bar{\gamma}_{z}$ being the average longitudinal Lorentz factor, and $h$ the harmonic number) with bandwidth $\Delta \omega /\left(h \omega_{r}\right) \ll 1$.

Moreover, for each particle in the beam, the relative deviation of the particles energy from $\gamma m_{e} c^{2}$ is small, i.e. $(\gamma-\bar{\gamma}) / \gamma \ll 1$. Therefore, in first approximation, we can neglect the difference between the average transverse 
velocity of electrons $\left\langle\vec{v}_{\perp}\right\rangle$ and $\vec{v}_{o \perp}$, so that $\vec{j}_{\perp} \equiv\left\langle\vec{v}_{\perp}\right\rangle \bar{\rho} \simeq$ $\vec{v}_{o \perp} \bar{\rho}$.

Furthermore, we will be interested in the total power emitted and in the directivity diagram of the radiation in the far zone. We therefore introduce the far zone approximation calling the observation angle $\vec{\theta}=\vec{r}_{\perp o} / z_{o}$ (see Fig (1), $z_{0}$ being the distance from the middle of the undulator to the observer. We also set $\theta \equiv|\vec{\theta}|$ and take the limit for $z_{o} \gg L_{w}$, where $L_{w}=N_{w} \lambda_{w}$ is the undulator length.

Finally, we introduce a coherent deflection angle, $\vec{\eta}^{(c)}$, to describe the transverse deflection of the electron beam as a whole [30]. We therefore perform the following substitution:

$$
{\overrightarrow{r^{\prime}}}_{o}(z) \longrightarrow \vec{r}^{(c)}\left(z, \vec{\eta}^{(c)}\right)={\overrightarrow{r^{\prime}}}_{o}(z)+\vec{\eta}^{(c)} z
$$

It follows that also $\vec{v}_{o}(z)$ should be substituted with $\vec{v}\left(z, \vec{\eta}^{(c)}\right)$, and that $\bar{\gamma}_{z}\left(z, \vec{\eta}^{(c)}\right)$ is now a function of both $z$ and $\vec{\eta}^{(c)}$. With these prescriptions, we obtain the following solution of Eq. (1):

$$
\begin{aligned}
& \quad \overrightarrow{\widetilde{E}}_{\perp}= \\
& -\frac{i \omega}{c z_{o}} \int_{-\infty}^{\infty} d z^{\prime} \int d \overrightarrow{l^{\prime}}\left(\frac{\vec{v}_{\perp}\left(z^{\prime}, \eta^{(c)}\right)}{c}-\vec{\theta}\right) \widetilde{\rho}\left(z^{\prime}, \overrightarrow{l^{\prime}}, \omega\right) \\
& \times \exp \left\{\frac{i \omega}{2 c}\left[z_{o} \theta^{2}-2 \vec{\theta} \cdot \vec{r}_{\perp}^{(c)}\left(z^{\prime}, \eta^{(c)}\right)-2 \vec{\theta} \cdot \vec{l}^{\prime}+z^{\prime} \theta^{2}\right]\right\} \\
& \times \exp \left\{+i \int_{0}^{z^{\prime}} d \bar{z} \frac{\omega}{2 c \bar{\gamma}_{z}^{2}\left(\bar{z}, \vec{\eta}^{(c)}\right)}\right\} .
\end{aligned}
$$

Substitution of the equations of motion Eq. (3) or Eq. (4), and of an appropriate expression for $\widetilde{\rho}$ into Eq. (6), yields the electric field in the far zone. We will consider a simple model where

$$
\begin{aligned}
& \widetilde{\rho}\left(z^{\prime}, \vec{l}^{\prime}, \omega\right)=\frac{(-e) N_{e} \bar{f}(\omega)}{2 \pi \sigma_{x} \sigma_{y}} \\
& \exp \left[-\frac{l_{x}^{\prime 2}}{2 \sigma_{x}^{2}}\right] \exp \left[-\frac{l_{y}^{\prime 2}}{2 \sigma_{y}^{2}}\right] \exp \left[i \frac{\omega}{c} \vec{\eta}^{(c)} \cdot \vec{l}^{\prime}\right],
\end{aligned}
$$

$\sigma_{x, y}$ being the electron beam rms transverse size, and $\bar{f}(\omega)$ the Fourier transform of the bunch temporal profile:

$$
f(t)=\frac{I(t)}{(-e) N_{e}}\left[1+b_{h} \cos \left(h \omega_{r} t\right)\right] .
$$

Here $b_{h}$ is the bunching at the $h$-th harmonic [31].

This model describes a bunched electron beam with Gaussian transverse profile, with current $I(t)$, longitudinally modulated at frequency $h \omega_{r}$. We are interested in a frequency range near the fundamental harmonic, $\omega_{r}$, or its $h$-th integer multiple. We specify how close the frequency $\omega$ is to the $h$-th harmonic by defining a detuning parameter $C_{h}$ :

$$
C_{h}=\frac{\omega}{2 \bar{\gamma}_{z}^{2} c}-h k_{w}=\frac{\Delta \omega}{\omega_{r}} k_{w}
$$

where $\omega=h \omega_{r}+\Delta \omega$. Whenever

$$
C_{h}+\frac{\omega}{2 c}\left[\left(\theta_{x}-\eta_{x}^{(c)}\right)^{2}+\left(\theta_{y}-\eta_{y}^{(c)}\right)^{2}\right] \ll k_{w},
$$

and $N_{w} \gg 1$, major simplifications arise because fast oscillating terms in powers of $\exp \left[i k_{w} z^{\prime}\right]$, which explicitly appear in Eq. (6) after substituting the trajectory [24], effectively average to zero after integration in $d z^{\prime}$. To complete the resonance approximation, we further select frequencies such that

$$
\frac{|\Delta \omega|}{\omega_{r}} \ll 1
$$

This condition on frequencies automatically selects the observation angles of interest: $h\left(\vec{\theta}-\vec{\eta}^{(c)}\right)^{2} \ll 1 / \bar{\gamma}_{z}^{2}$. Under the constraint imposed by (11), independently of the value of $K$, we obtain from Eq. [6] analytical expressions for the angular spectral fluxes [32]. These expressions describe the coherent emission in the far zone from modulated electron beams in planar or helical undulators, and strongly depend on the Fresnel parameter $N_{x, y}$ defined as

$$
N_{x, y}=\frac{h \omega_{r} \sigma_{x, y}^{2}}{c L_{w}}
$$

In the helical case, the spectral flux at the $h$-th harmonic is given by:

$$
\begin{aligned}
& \frac{d P_{h}}{d \Omega}=\frac{\pi}{c}\left[\frac{b_{h} I(t) h N_{w} K \bar{\gamma}_{z}}{((h-1) !)\left(1+K^{2}\right)}\right]^{2} \\
& {\left[\frac{h^{2} L_{w} K^{2} \omega_{r}}{4 \pi N_{w} c\left(1+K^{2}\right)}\left(\theta_{x}^{2}+\theta_{y}^{2}\right)\right]^{h-1}} \\
& \times \operatorname{sinc}^{2}\left[\frac{h L_{w} \omega_{r}}{4 c}\left(\theta_{x}^{2}+\theta_{y}^{2}\right)\right] \\
& \times \exp \left[-\frac{h L_{w} \omega_{r}}{c}\left(N_{x} \theta_{x}^{2}+N_{y} \theta_{y}^{2}\right)\right] .
\end{aligned}
$$

Figures 2 and 3 show the angular spectral flux as obtained from Eq. (13) for the CHG and NHG, respectively. For the calculation, use has been made of the parameters reported in Table $\square$, corresponding to the experimental setup described in the following Section. 


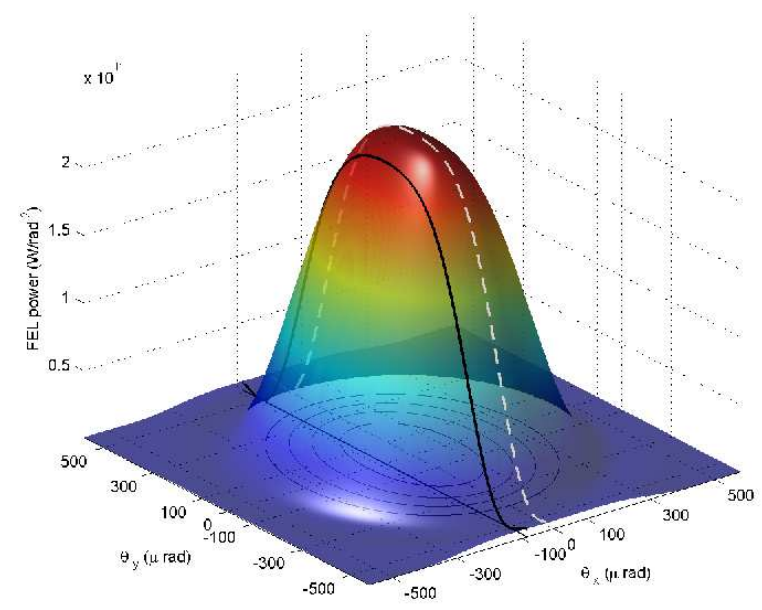

FIG. 2: Angular spectral flux in CHG mode, as predicted by Eq. (13). For the calculation, use has been made of the parameters reported in Table प corresponding to the case of the Elettra storage-ring FEL. In this case, light is emitted at the radiator fundamental wavelength (i.e., $h=1$, corresponding to $260 \mathrm{~nm}$ ), which is the third harmonic of the seed wavelength $(780 \mathrm{~nm})$. .

\section{EXPERIMENTAL SETUP}

The predictions of the model presented in the previous Section have been tested on the Elettra storage-ring FEL, whose scheme is shown in Fig. 4. The setup, which is based on two independent APPLE II undulators separated by a magnetic chicane, is very flexible: depending on the undulator configuration, both NHG and CHG schemes can be implemented. The laser pulse emitted by a Ti:sapphire laser (fundamental wavelength at 780 $\mathrm{nm}$ ) interacts with the electron bunch within the modulator, inducing an energy modulation in the electron beam. After the conversion of the energy modulation into spatial bunching, occurring into the magnetic chicane, the bunch enters the radiator and emits coherent radiation at one of the harmonics of the seed laser (the third one, i.e. $260 \mathrm{~nm}$, for the experiments presented in this paper). The harmonic radiation is first monochromatized and then acquired on a digital oscilloscope by means of a photomultiplier tube (PMT), see Fig. 4. The latter is fast enough to resolve the pulsed dynamics of the electron bunch photoemission (nanonosecond scale), but does not allow to resolve the photon pulse shape (tens of ps). A diaphragm of $1.4 \mathrm{~mm}$ is placed downstream the radiator, before the detector. The diaphragm is used to select only the portion of radiation emitted within a small cone close to the undulator axis (angular acceptance of about $100 \mu \mathrm{rad})$. By moving the diaphragm, it is possible to characterize the angular distribution of the produced coherent radiation.

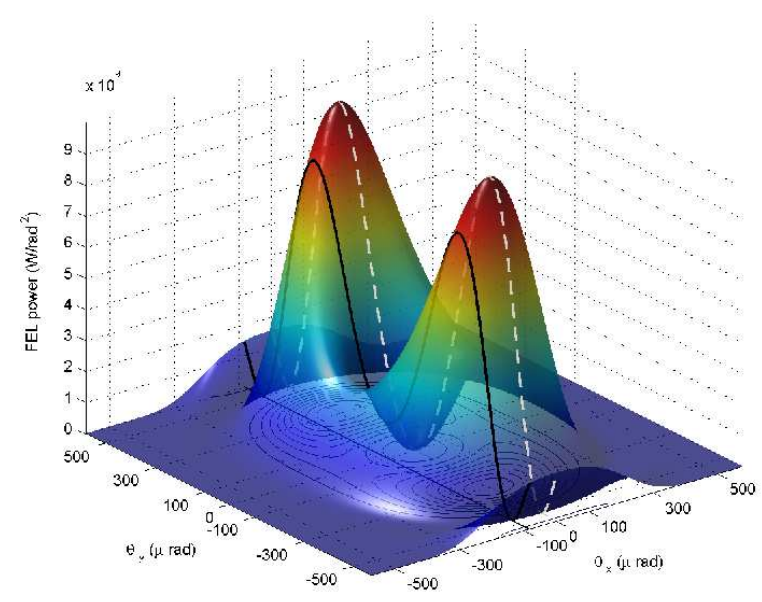

FIG. 3: Angular spectral flux in NHG mode, as predicted by Eq. (13). For the calculation, use has been made of parameters reported in Table [. corresponding to the case of the Elettra storage-ring FEL. In this case, light is emitted at the second harmonic of the radiator fundamental wavelength (i.e., $h=2$, corresponding to $260 \mathrm{~nm}$ ), which is the third harmonic of the seed wavelength $(780 \mathrm{~nm})$.

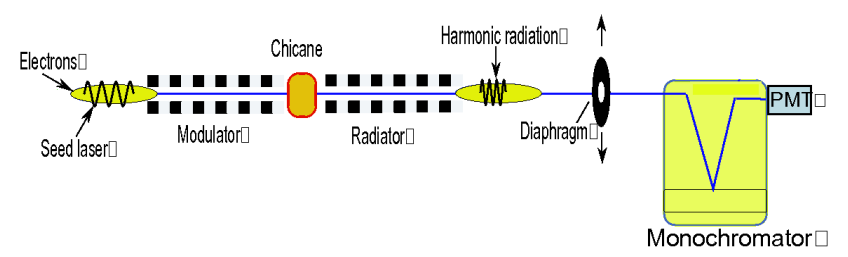

FIG. 4: Schematic layout of the Elettra storage-ring FEL.

For the reported experiments, Elettra was operated in single-bunch mode at $0.75 \mathrm{GeV}(\gamma \simeq 1468)$, the peak bunch current and its revolution period being, respectively, $\simeq 10 \mathrm{~A}$ and $864 \mathrm{~ns}$. The seed repetition rate is $300 \mathrm{~Hz}$. The laser pulse and the electron bunch duration are, respectively, $120 \mathrm{fs}$ (rms) and about $35 \mathrm{ps}$ (rms). A stable laser-electron synchronization with a jitter of few ps 26] guarantees an efficient and stable seeding process, with shot-to-shot fluctuations of the order of few percent. The main experimental parameters, also used to obtain the theoretical angular spectral flux reported in Figs. 2 and 3, are reported in Table \. More detailed information about the experimental setup, as well as about the performance of the Elettra storage-ring FEL, can be found in [26].

\section{RESULTS}

In this Section we present the experimental data and perform a quantitative comparison between experiments 
TABLE I: Main experimental parameters.

\begin{tabular}{lll}
\hline \hline Electron beam & & \\
\hline Peak current & 10 & $\mathrm{~A}$ \\
Normaized energy $(\gamma)$ & 35 & $\mathrm{ps}$ \\
Bunch length $(\mathrm{rms})$ & $\simeq 0.1$ & $\%$ \\
Relative energy spread & 0.6 & \\
Bunching $\left(b_{h}\right)$ & 200 & $\mu \mathrm{m}$ \\
Horizontal transverse size $(\mathrm{rms})\left(\sigma_{x}\right)$ & 10 & $\mu \mathrm{m}$ \\
Vertical transverse size $(\mathrm{rms})\left(\sigma_{y}\right)$ & & $\mathrm{GW}$ \\
\hline Seed laser & $\sim 10$ & $\mathrm{~nm}$ \\
\hline Power & 780 & $\mathrm{fs}$ \\
Wavelength & $\sim 120$ & \\
Pulse length $($ rms $)$ & Horiz., Circ. & \\
Polarization & & $\mathrm{m}$ \\
\hline Radiator & 0.10 & $\mathrm{~nm}$ \\
\hline Periodicity & 20 & $\mathrm{~nm}$ \\
Number of periods $\left(N_{w}\right)$ & & \\
Resonant wavelength $\left(\frac{2 \pi c}{\omega_{r}}\right)$, CHG mode & 260 & \\
Undulator parameter $(K)$ in CHG mode & 3.19 & $2 \pi c$ \\
Resonant wavelength $\left(\frac{2 \pi}{\omega_{r}}\right)$, NHG mode & 520 & \\
Undulator parameter $(K)$ in NHG mode & 4.63 & \\
Polarization & Horiz, Circ. & \\
\hline \hline
\end{tabular}

and theory.

A first set of measurements has been carried out in CHG configuration, tuning the radiator at $260 \mathrm{~nm}$ (third harmonic of the seed wavelength), in circular polarization. The intensity detected by the PMT, as a function of $\theta_{y}$, is represented by the open circles in Fig. 5. As expected (see Fig. 21), the intensity has a maximum in correspondence of the undulator axis $\left(\theta_{y}=0\right)$.

Due to some mechanical limitations intrinsic to our device, the diaphragm can be moved only downstream along the vertical direction. As a consequence, no measurements have been performed along positive $\theta_{y}$ 's, nor along the $\theta_{x}$ direction. Error bars represent the result of several independent measurements. Fluctuations are mainly due to FEL instabilities and to possible undesired slight shifts of the electron-beam trajectory in the time period between successive measurements. Data have been acquired at quite low average electron-beam current $(<1 \mathrm{~mA})$, that is in a regime characterized by relatively long beam lifetimes (order of hours). However, since the harmonic intensity varies as the square of the number of charges involved into the emission process [25], even small current variations may reflect in significant intensity fluctuations. In order to take this effect into account, a correction factor has been applied to experimental data. In Fig. 5, continuous and dotted lines represent the theoretical predictions obtained using Eq. (13) and the parameters reported in Table I. The dotted line has been obtained by integrating the curve shown

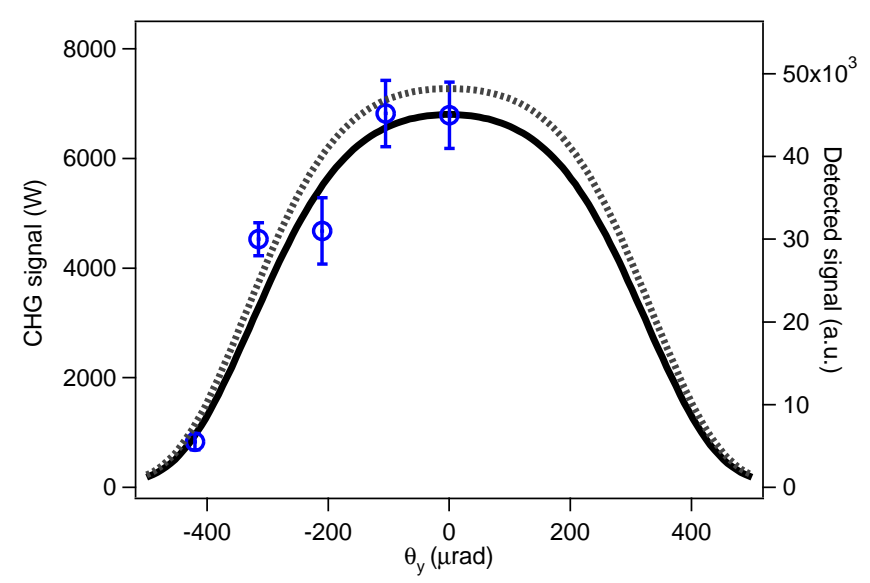

FIG. 5: Intensity of the CHG signal at $260 \mathrm{~nm}$, third harmonic of the seed wavelength, as a function of the angle $\theta_{y}$ (vertical direction, see Fig. 2) between the undulator axis and the line from the center of the ondulator to the position of the diaphragm. Open circles: measurements (right vertical scale). Continuous and dotted lines: theoretical predictions (left vertical scale) obtained using Eq. (13) and the set of parameters reported in Table I Dotted line: integration of the curve shown in Fig 2 along $\theta_{y}$, over an angle of $100 \mu$ rad around $\theta_{x}=0$. In plotting experimental data, a correction factor has been used to take into account (slight) current variations occurred in the time period between successive measurements.

in Fig. 2 along $\theta_{y}$, over an angle of $100 \mu \mathrm{rad}$ (the diaphragm acceptance) around $\theta_{x}=0$ (corresponding to a perfect horizontal alignment between the electro $n$ beam and the undulator axis). The continuous line has been instead obtained by integrating the curve shown in Fig. 2 along $\theta_{y}$, over an angle of $100 \mu \mathrm{rad}$ around $\theta_{x}=80 \mu \mathrm{rad}$. The latter value corresponds to the best possible experimental accuracy in controlling the horizontal alignment between the electron beam and the undulator axis.

The same (experimental and theoretical) analysis has been carried out in NHG configuration. In order to reproduce as much as possible the experimental conditions of the CHG configuration, the radiator has been tuned at $520 \mathrm{~nm}$ (in circular polarization). This avoids any seedelectron interaction inside the radiator and, as a consequence, prevents any undesired degradation of the beam quality (i.e., an increase of the beam energy spread).

Since $520 \mathrm{~nm}$ is not a harmonic of the input seed wavelength, no bunching is produced in the modulator at such wavelength. As a consequence, there is no coherent emission at the fundamental wavelength of the radiator. However, one obtains coherent emission through NHG at 260 $\mathrm{nm}$ and $130 \mathrm{~nm}$ (second and fourth harmonics, respectively, of the radiator wavelength). As for the case of CHG, the intensity of the coherent emission at $260 \mathrm{~nm}$ is detected using the PMT. Results are reported in Fig. 6. As expected (see Fig. 3), due to the finite aperture of the diaphragm, NHG emission is not exactly zero on axis.

In order to check the agreement between measurements 


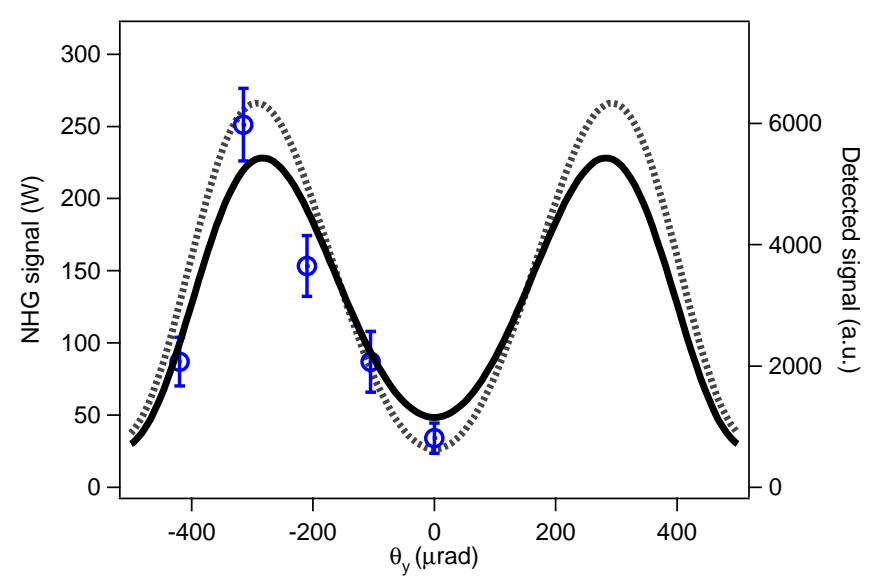

FIG. 6: Intensity of NHG at $260 \mathrm{~nm}$, second harmonic of the radiator fundamental wavelength, as a function of the angle $\theta_{y}$. Open circles: measurements (right vertical scale). Continuous and dotted lines: theoretical predictions (left vertical scale) obtained using Eq. 13) and the set of parameters reported in Table I] Dotted line: cut of Fig. 3 along $\theta_{y}$, for $\theta_{x}=0$; continuous line: integration of the curve shown in Fig 2 along $\theta_{y}$, over an angle of $100 \mu \mathrm{rad}$ around $\theta_{x}=80$ $\mu \mathrm{rad}$. In plotting experimental data, a correction factor has been used to take into account (slight) current variations occurred in the time period between successive measurements.

and theory, one can compare the experimental and theoretical ratios between the peak $\mathrm{CHG}$ and residual NHG on-axis intensities. Using the data reported in Figs. [5 and 66, and considering the (more realistic) theoretical continuous curves, one gets an experimental ratio of about 60 and a theoretical one of 140. Taking into account the above-mentioned experimental incertitude, as well as the approximations intrinsic to the theoretical model, the agreement can be considered satisfactory [33].

\section{CONCLUSIONS}

We performed a quantitative analysis of the angular distribution of Nonlinear Harmonic Generation in heli- cal undulators. Measurements carried out on the Elettra storage-ring free-electron laser, have been compared to the predictions from a model based on the analytical solution of the paraxial Maxwell equations. As we have shown, the agreement can be considered as satisfactory. This result allows one to consider the theoretical model as a reliable tool for predicting the off-axis photon flux of next-generation FELs, operated in Nonlinear Harmonic Generation mode.

In fact, predictions are in general quite pessimistic. As an example, calculations have been recently performed [27] for harmonic off-axis emission in the case of the FERMI@Elettra FEL [28], an FEL user facility presently under commissioning at Sincrotrone Trieste. In the case of FERMI, operating at $5 \mathrm{~nm}$ in circular polarization, FEL saturation is easily reached and about $3 \cdot 10^{12}$ on-axis photons/pulse at the fundamental wavelength are produced. The flux reduction at the second (off-axis) harmonic, calculated using the theoretical model, is drastic: only about $10^{8}$ photons/pulse are expected at $2.5 \mathrm{~nm}$. An additional factor of about $\cdot 10^{4}$ is lost if considering the third harmonic emission at $1.33 \mathrm{~nm}$ [27]. Having provided an experimental validation of the theoretical model, we can conclude that collecting off-axis emission does not allow to recover a significant photon flux when the FEL is operated in Nonlinear Harmonic Generation mode, with circularly polarized undulators.

As an alternative method to obtain significant (onaxis) circularly polarized harmonic radiation, one may consider the possibility to operate the radiator in elliptically polarized mode. Such a method gave interesting results in the case of spontaneous (linear) harmonic radiation [29]. As a future study, we plan to investigate its efficiency when applied to Nonlinear Harmonic Generation in FEL's.

\section{ACKNOWLEDGEMENT}

We acknowledge useful discussions W. M. Fawley. We also thanks the Elettra machine group for the technical support.
[1] F. Meier and D. Pescia, Phys. Rev. Lett. 47, 374 (2001).

[2] J. P. Hannon et al., Phys. Rev. Lett. 61, 1245 (1988).

[3] P. Carra, M. Altarelli and Franois de Bergevin, Phys. Rev. B 40, 7324 (1989).

[4] J. C. Sutherland et al., Nucl. Instrum. Methods Phys. Res. A 172, 195 (1994).

[5] M. Blume and D. Gibbs, Phys. Rev. B 37, 1779 (1988).

[6] R. Neutze et al., Nature 406757 (2000); W. A. Barletta and H. Winck, Nucl. Instr. and Meth. A 5001 (2003); N. Gedik et al., Science 316425 (2007); A. Cavalieri, Nature 448651 (2007). J.T.Costello, Journal of Physics: Conference Series 88012057 (2007) and references therein,
J. Kirz, Nature Physics 2799 (2006), H. N. Chapman et al., Nature 448676 (2007).

[7] M.J. Schmitt and C.J. Elliott, Phys. Rev. A 34, 4843 (1986).

[8] R. Bonifacio, L. De Salvo and P. Pierini, Nucl. Instrum. and Meth. A 293, 627 (1990).

[9] G. Dattoli and G. Voykov, Phys. Rev. E 48, 3030 (1993).

[10] Z. Huang and K.J. Kim, Phys. Rev. E 62, 7295-7308 (2000).

[11] S.G. Biedron et al., Phys. Rev. ST AB 5, 030701 (2002).

[12] H.P. Freund, G.P. O'Shea and S.G. Biedron, Phys. Rev. Lett. 94, 074802 (2005). 
[13] E.L. Saldin, E.A. Schneidmiller and M.V. Yurkov, Phys. Rev. ST AB 9, 030702 (2006).

[14] M. Labat et al., Proceeding FEL Conference 2009 (http://accelconf.web.cern.ch/AccelConf/ FEL2009/papers/wepc56.pdf).

[15] A. Kondratenko and E. Saldin, Part. Accel. 10, 207 (1980); Y. Derbenev, A. Kondratenko, and E. Saldin, Nucl. Instrum. and Meth. A193 415 (1982); R. Bonifacio, C. Pellegrini, and L. Narducci, Opt. Commun. 50, 373 (1984); M. Babzien et al., Phys. Rev. E. 576093 (1998); S. V. Milton, Phys. Rev. Lett. 85988 (2000); J. Andruszkow, Phys. Rev. Lett. 853825 (2000); R. Brinkmann, Proceedings FEL Conference 2006.

[16] A. Doyuran et al., Phys. Rev. Lett. 865902 (2001).

[17] L.H. Yu et al., Phys. Rev. Lett. 91074801 (2003).

[18] T. Pfeiffer et al., Rep. Prog. Phys. 69443 (2006); J. Seres at al., Nature physics 3878 (2007).

[19] E. Allaria et al., Phys. Rev. Lett. 100174801 (2008).

[20] G. Geloni, E. Saldin, E. Schneidmiller and M. Yurkov, Opt. Comm. 271, 207 (2007).

[21] G. Geloni, E. Saldin, E. Schneidmiller, M. Yurkov, Opt. Comm. 276, 167 (2007).

[22] G. Geloni, E. Saldin, E. Schneidmiller, M. Yurkov, Nucl. Instrum. Methods Phys. Res. A 584, 219 (2008).
[23] G. Geloni, E. Saldin, E. Schneidmiller, M. Yurkov, Nucl. Instrum. Methods Phys. Res. A 581, 856 (2007).

[24] D. Alferov, Y. Bashmakov and E. Bessonov, Sov. Phys. Tech. Phys. 18, 1336 (1974).

[25] G. De Ninno et al., Phys. Rev. Lett. 101053902 (2008).

[26] C. Spezzani et al., Nucl. Instrum. Methods Phys. Res. A 596451 (2008).

[27] W. Fawley, private communication.

[28] C. Bocchetta et al., FERMI@Elettra Conceptual Design Report, available at ttp://www.elettra.trieste.it/FERMI.

[29] S. Khan et al., Phys. Rev. Lett. 97074801 (2006).

[30] With this, we assume that the deflection angle $\vec{\eta}^{(c)}$ is constant. This is the case only if we do not account for focusing elements within the undulator.

[31] Note that this definition of bunching gives a coefficient which is a factor two larger than the one used in other works (see, e.g., [17]).

[32] In CGS units. Conversion to SI units is done by dividing by a factor of $10^{-7}$.

[33] The agreement becomes worse if the less realistic dashed theoretical curves in Figs. 5 and 6 are used. In this case, one obtains a theoretical ratio of about 280 . 